\title{
Doppler Tomography of Accretion Disks and Streams in Close Binaries
}

\author{
Mercedes T. Richards \\ Department of Astronomy \& Astrophysics, Pennsylvania State University, 525 Davey \\ Laboratory, University Park, PA, 16802, USA \\ email: mrichards@astro.psu.edu
}

\begin{abstract}
The application of tomography to the study of gas flows in interacting binaries has led to fascinating images of the Cataclysmic Variables and Algol-type binaries. Such detailed images are currently unachievable using direct-imaging techniques. Numerous images of accretion flows have now been derived from optical and ultraviolet spectra and they have been used to identify multiple emission sources including the gas stream, accretion disk, accretion annulus, shock regions, and the chromosphere of the mass loser. It was difficult to distinguish between the separate sources of emission since these sources have overlapping velocities in the Doppler tomogram. However, with the aid of a new spectral synthesis code, we can now systematically extract the individual emission sources to sequentially isolate the images of the disk and gas stream. With these new tools, we have begun to extract the critical properties of the disk and gas stream more accurately than previously possible.
\end{abstract}

Keywords. accretion, accretion disks, stars: activity, (stars:) binaries: eclipsing, hydrodynamics, radiative transfer, (stars:) novae, cataclysmic variables

\section{Introduction}

Since the technique of Doppler tomography (Marsh \& Horne 1988) was introduced 18 years ago, it has been used to provide indirect images of accretion structures in close binaries which cannot be resolved spatially with the largest telescopes. The only direct images of distinct gas flows between stars in an interacting binary were produced in 2005 by Karovska et al. (2005) from soft X-ray Chandra and HST images of Mira. In all other cases, we have to resort to indirect techniques to study the emission sources and gas flows in the binary. The more general technique of tomography has been used successfully in medicine, geophysics, archaeology, and oceanography to construct threedimensional (3D) images from two-dimensional (2D) pictures or "slices" through the object collected at many positions around the object. These slices or projections are represented by the Radon transform (Radon 1917); the 3D image is recovered through a summation process called back projection; and the overall image reconstruction procedure is known as tomography. In astronomy, the technique can be readily applied to eclipsing binaries and rotating stars, which provide changing views of the system, and the process is called Doppler Tomography because the gas motions detected through Doppler shifts provide an image of the accretion flows in velocity coordinates.

Doppler tomography has revitalized the study of accretion structures in interacting binaries which contain both compact and normal mass gainers. This procedure has been described in several papers: Marsh \& Horne (1988), Robinson, Marsh, \& Smak (1993), Kaitchuck et al. (1994), Staude et al. (2001), Richards (2001), and Richards (2004). The technique has now been extended to the third dimension by Agafonov et al. (2006) to reveal the gas flows beyond the orbital plane. To date, there have been two conferences on the application of tomography to astronomy: "Astrotomography: An International 
Workshop on Indirect Imaging," held in Brussels in 2000 July, published in Lecture Notes in Physics, Vol. 573 (edited by H.M.J. Boffin, D. Steeghs, \& J. Cuypers), and Joint Discussion No. 9 on "Astrotomography" at the IAU General Assembly in Sydney, in 2003 July, which was published in Astronomische Nachrichten, Vol. 325, No. 3 (2004). This latter volume provides a very good summary of the various astrotomography techniques.

The basic technique of tomography introduced by Johann Radon (Radon 1917; Herman 1980; Shepp 1983) can be used to create an image of an object from projections. In the most common application, if a $2 \mathrm{D}$ object is described by the function $f(x, y)$, then the Radon transform of that $2 \mathrm{D}$ object is a set of $1 \mathrm{D}$ projections along the line $s=$ $x \cos \theta+y \sin \theta$ for the full range of angles $\theta$ :

$$
p(s, \theta)=\int_{-\infty}^{\infty} \int_{-\infty}^{\infty} f(x, y) \delta(x \cos \theta+y \sin \theta-s) d x d y
$$

where, $\delta$ is the Dirac delta function such that $\delta(a)=\infty$ if $a=0$, and $\delta(a)=0$, otherwise. The technique of back projection is the process in which this $1 \mathrm{D}$ projection is inverted to reveal the $2 \mathrm{D}$ function $f(x, y)$. This is fairly easy to do since the $1 \mathrm{D}$ Fourier transform of the projection function $p(s, \theta)$ is equal to the 2D Fourier transform of the 2D object, $f(x, y)$, calculated along the projection line. The result is

$$
f(x, y)=\int_{0}^{2 \pi} \int_{-\infty}^{\infty} \int_{-\infty}^{\infty} p(s, \theta)|\omega| e^{2 \pi i \omega(x \cos \theta+y \cos \theta-s)} d s d \omega d \theta .
$$

Similarly, in the standard two-dimensional case of Doppler tomography, the Radon transform of the function, $I=f\left(v_{x}, v_{y}\right)$, is a set of $1 \mathrm{D}$ projections, $p\left(v_{r}, \phi\right)$, which is the line profile at each orbital phase, $\phi$. The $2 \mathrm{D}$ function is obtained by integrating over the line: $v_{r}=v_{x} \cos \phi+v_{y} \sin \phi$ and with a change of variables from the Cartesian to the velocity frame: $x \rightarrow v_{x}, y \rightarrow v_{y}, s \rightarrow v_{r}, \theta \rightarrow \phi$.

$$
f\left(v_{x}, v_{y}\right)=\int_{0}^{2 \pi} \int_{-\infty}^{\infty} \int_{-\infty}^{\infty} p\left(v_{r}, \phi\right)|\omega| e^{2 \pi i \omega\left(v_{x} \cos \phi+v_{y} \sin \phi-v_{r}\right)} d v_{r} d \omega d \phi
$$

This change of variables produces the same formulation as the filtered back projection method derived for Doppler tomography (see Marsh \& Horne 1988, Robinson et al. 1993, and Kaitchuck et al. 1994). This process can be readily extended to the case of the 3D tomogram, since the Radon transform of $f(x, y, z)$, is a set of $2 \mathrm{D}$ projections, $p(s, \theta, \psi)$.

\section{Application to Compact Binaries and Algol-type Binaries}

Accretion disks are found in binaries which contain white dwarfs and neutron stars (e.g., cataclysmic variables $(\mathrm{CVs})$ and soft X-ray binaries), and non-compact main sequence stars (e.g., Algol-type binaries). The main differences between these systems are: (1) mass transfer occurs onto a compact object in the CVs and X-ray binaries, while the mass gainer in the Algols is a main sequence star; (2) the accretion structures in the compact systems are bright relative to the stars, while the structures in the Algols are faint relative to the luminous main sequence primary star; (3) the large size of the mass gainer in the Algols leads to the direct impact of the gas stream onto the stellar photosphere in the short-period Algols ( $P_{\text {orb }} \leqslant 5$ days), while this type of impact does not occur in the long-period Algols $\left(P_{\text {orb }}>5-6\right.$ days $)$ or the compact systems. The result is that a complex set of accretion structures is formed in the short-period Algols compared to the classical accretion disks in the other systems. 


\subsection{Images of Cataclysmic Variables and X-ray Binaries}

The non-magnetic CVs (e.g., dwarf novae) contain a white dwarf with no field or a weak magnetic field, the magnetic CVs (or polars) have very strong magnetic fields which inhibit the formation of an accretion disk, and the intermediate group of intermediate polars contains a white dwarf with a moderate magnetic field. Images of over $33 \mathrm{CVs}$ in outburst and quiescent states have now been produced (see reviews by Marsh 2001, Morales-Rueda 2004, Steeghs 2004, Schwope et al. 2004). These include 18 dwarf novae (e.g., U Gem, WZ Sge, IP Peg), 2 intermediate polars (EX Hya, V1025 Cen), 9 polars (e.g., AM Her, UZ For, V1309 Ori), 2 old novae (V841 Oph, RR Pic), and 2 helium CVs (AM CVn, GP Com). The complete list is given in Morales-Rueda (2004). Tomograms have been produced of spectra at the following wavelengths: $\mathrm{H} \alpha, \mathrm{H} \beta, \mathrm{H} \gamma, \mathrm{H} \delta$, $\mathrm{He} \mathrm{I} \lambda 4472$ $\AA$, He I $\lambda 5876 \AA$, He I $\lambda 6678 \AA$, He I $\lambda 8236 \AA$, He I $\lambda 1.083 \mu$, Na I, Ca II, He II $\lambda 4686$ $\AA$, and He II $\lambda 1.163 \mu$.

The tomograms of non-magnetic CVs are usually dominated by the image of the accretion disk, with less prominent gas streams. It was in these images that spiral structure in disks was first discovered (e.g., Steeghs et al. 1997, Harlaftis et al. 1999, Morales-Rueda 2000, Groot 2001). A movie showing the changes in the disk structure from quiescent to outburst states in WZ Sge was made by Steeghs (2004) using multiple He II and H $\beta$ tomograms. Hydrodynamic simulations of these systems by Sawada et al. (1986) had earlier proposed that the spiral structure was produced by tidally-induced spiral density wave. These simulations have been refined by Bisikalo \& Matsuda (2007).

The magnetic CVs have provided the most impressive images of accretion streams (e.g., HU Aqr: Schwope et al. 1997; V1309 Ori: Staude et al. 2001). In these systems, the strong magnetic field inhibits the formation of an accretion disk, so the gas stream dominates the image. Ultraviolet and X-ray images of two X-ray binaries, Her X-1 and SMC X-1, have been produced (see review by Vrtilek et al. 2004) and suggest that there are still too few studies to make a coherent picture of the accretion structures in these systems. Her X-1 displays a prominent accretion disk, while most of the emission in the massive wind-fed system SMC X-1 is associated with the mass losing star.

\subsection{Images of the Algols}

Doppler tomograms of 12 eclipsing Algols, 1 non-eclipsing Algol (CX Dra), and 1 RS CVn binary have been produced (see reviews by Richards 2001 and Richards 2004). The RS CVn binary V711 Tau (HR 1099) was included to illustrate the location and appearance of chromospheric $\mathrm{H} \alpha$ emission in the tomogram since this binery was not predicted to be in the process of Roche lobe overflow. The images of the Algols were made from over 2500 spectra collected systematically from 1992 - 2004: RZ Cas, $\delta$ Lib, RW Tau, $\beta$ Per (Algol), TX UMa, U Sge, S Equ, U CrB, RS Vul, SW Cyg, CX Dra, TT Hya, AU Mon, in order of increasing orbital period, $P_{\text {orb }}=1.2-11.1$ days (Richards 2004). Most of the tomograms are based on optical spectra at the following wavelengths: $\mathrm{H} \alpha, \mathrm{H} \beta$, $\mathrm{He} \mathrm{I}$ $(6678 \AA)$ and Si II (6371 $\AA$ ). In the ultraviolet, a tomogram of U Sge was produced from the Si IV (1394 A) line (Kempner \& Richards 1999), while MgII h \& k lines were used to image the chromosphere in V711 Tau (Richards \& Rosolowsky 1998).

The greatest challenge in the study of accretion structure in the Algols is that these structures are faint relative to the luminous primary star. So, in order to extract information about these structures, it is necessary to compute the stellar contribution to the line profile and remove it from the observed spectra (Richards 1993). This procedure assumes that the accretion structure is optically thin; an assumption that has been tested successfully in the case of long-period systems where tomograms can be derived from both 
the observed and difference spectra. The resulting difference profiles display enhanced emission profiles which can readily be processed into Doppler images.

The $H \alpha$ tomograms show that the Algols display several sources of emission in the orbital plane (Richards 2001, Richards 2004): (1) the gas stream along the predicted gravitational trajectory (Richards et al. 1995); (2) transient or permanent accretion disks (Richards et al. 1995, Albright \& Richards 1996); (3) a star-stream impact region where the gas stream strikes the stellar photosphere; (4) a disk-stream impact region where the disk strikes the incoming gas stream; (5) a circumprimary bulge produced by the impact of the high velocity gas stream onto the slowly rotating photosphere; (6) the chromosphere of the secondary (Richards \& Albright 1996); and (7) an absorption zone that overlaps with the locus of hotter gas seen in the UV tomogram (Kempner \& Richards 1999).

The gas stream emission detected in the Algols represented the first ever images of these structures for the entire class of interacting binaries. Later, even more impressive gas streams were found in polars by Schwope et al. (1997). The comprehensive study of the multiwavelength tomograms of the non-eclipsing system of CX Dra (Richards et al. 2000) demonstrated that multiwavelength tomograms could be used effectively to derive the properties of the accretion structures in the binary; including a well-formed disk (derived from He I and Si II spectra) and a truncated gas stream (derived from the H $\alpha$ and $\mathrm{H} \beta$ lines). Moreover, multi-epoch tomograms have shown that the emission sources in systems like $\beta$ Per and TX UMa are quite stable; while other systems like U Sge and U CrB display structures which alternate between stream-like and disk-like states. The hydrodynamic simulations of Richards \& Ratliff (1998) suggested that this variability was caused by changes in the mass transfer rate over intervals within a few orbital periods.

\section{Separation of the Emission Sources Using Synthetic Spectra}

The complex emission sources in the Algols cannot readily be separated into the individual components because the various sources overlap in the velocity domain. In order to study these components, Budaj \& Richards (2004) developed a spectrum synthesis code called SHELLSPEC. Synthetic spectra were calculated to sequentially model (1) the stars, (2) the stars and disk, and (3) the stars, disk, and gas stream combined. Doppler tomograms were produced by incorporating these three sets of synthetic spectra. The technique was illustrated in the case of the long-period Algol, TT Hya $\left(P_{\text {orb }}=6.96\right.$ days) by Budaj et al. (2005) and Miller et al. (2007).

\subsection{The Synthetic Spectra}

The SHELLSPEC code solves in LTE the simple radiative transfer along the line of sight in an optional optically thin 3D moving medium (Budaj \& Richards 2004). Transparent (or non-transparent) objects such as a spot, disk, stream, jet, shell, or stars may be defined (or embedded) in three dimensions and their composite synthetic spectrum calculated (Budaj \& Richards 2004, Budaj et al. 2005). The stars may have Roche geometry and known intrinsic spectra. The scattered light from a central object can be taken into account assuming an optically thin environment. Intrinsic spectra (intensity or flux from a unit surface area not broadened by the rotation) of the primary and secondary star can be precalculated using the codes TLUSTY and SYNSPEC, (Hubeny 1998, Hubeny \& Lanz 1992, Hubeny \& Lanz 1995, Hubeny et al. 1994) as well as with the Kurucz (1993) model atmospheres code. These intrinsic spectra can be assigned to the primary and secondary star and a complex spectrum of both stars and the circumstellar matter can 

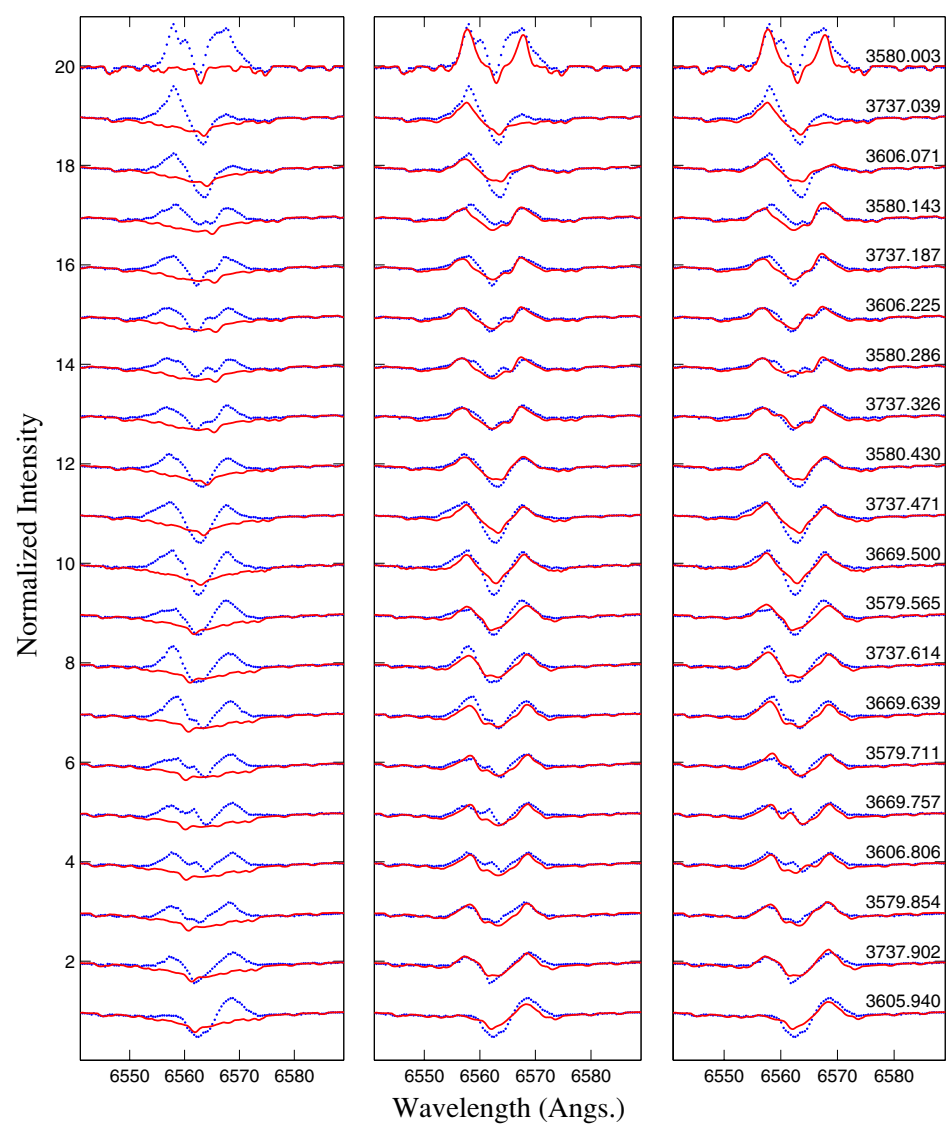

Figure 1. Comparison of the observed (dotted line) and synthetic (solid line) $\mathrm{H} \alpha$ spectra of TT Hya. The epochs and orbital phases are listed to the right of the figure. The synthetic spectra include calculations of (a) stars only (left frame); (b) stars and disk (middle frame); and (c) stars, disk, and gas stream (right frame). There is a significant improvement in the fit between the observed and synthetic spectra once the accretion disk is included, but only a minor improvement once the gas stream is included in the model (courtesy: Miller et al. 2007).

be calculated using SHELlSPEC. Synthetic light curves or trailing spectrograms can be produced by changing the view points on the $3 \mathrm{D}$ object.

The SHELlsPec code was first applied to the long-period Algol, TT Hya. Budaj et al. (2005) compared observed and synthetic $\mathrm{H} \alpha$ spectra and found that the disk extends to about 10 solar radii in the orbital plane and has a vertical thickness comparable to the diameter of the primary star. They also found that the overall strength of the emission is regulated mainly by the density and temperature of the disk, while the position and separation of the emission peaks are influenced by the outer disk radius, the inclination, and the radial density profile. In addition, the depth of the central absorption is very sensitive to the temperature, inclination, geometry, and dynamics of the disk. Further analyses of the optical and ultraviolet spectra of TT Hya were used by Miller et al. (2007) to refine the properties of the disk. These refined disk and system properties were used to generate synthetic spectra of the disk for input in the tomography code.

For TT Hya, the assumed temperature of the disk was $T=7000 \mathrm{~K}$ and the disk density at the inner radius was $3.3 \times 10^{-14} \mathrm{~g} \mathrm{~cm}^{-3}$ (Miller et al. 2007). The initial gas stream temperature was set to $T=8000 \mathrm{~K}$ to correspond to the peak in $\mathrm{H} \alpha$ emissivity. 
The estimated density of the stream at the starting point $\left(2 \times 10^{-14} \mathrm{~g} \mathrm{~cm}^{-3}\right)$ was chosen to improve the fit between the observed and synthetic emission at the quadratures of the orbit and during the eclipses. For these calculations, the density was allowed to vary along the stream to satisfy the continuity equation. The electron number densities in the disk and stream were calculated from the temperature, density, and chemical composition using the Newton-Raphson linearization method. Miller et al. (2007) obtained a mass transfer rate of $\sim 2 \times 10^{-10} M_{\odot} y r^{-1}$, based on the velocity, density, and cross-section of the gas stream above and below the disk. This rate is two orders of magnitude higher than the lower limit of $10^{-12} M_{\odot} y r^{-1}$ obtained by Peters \& Polidan (1998).

\subsection{Extraction of the Disk and Stream and Discovery of an Eccentric Disk}

The observed $\mathrm{H} \alpha$ spectra of TT Hya were compared with synthetic spectra of (1) the stars only, (2) stars and disk, and (3) stars, disk, and stream. Figure 1 illustrates that the stellar spectrum is not sufficient to explain the observed profile, but the inclusion of the disk emission made a remarkable improvement to the fit between observed and synthetic spectra. The inclusion of the gas stream only slightly improves the fit, especially near the quadratures.

To illustrate the quality of these fits, Miller et al. (2007) generated tomograms from (a) the observed spectra, (b) the observed spectra minus the synthetic stellar spectra, (c) the observed spectra minus the synthetic spectra of the stars and accretion disk, and (d) the observed spectra minus the synthetic spectra of the stars, disk, and gas stream. H $\alpha$ spectra of TT Hya spanning 16 years from 1985-2001 were used to make the images. The resulting tomograms are shown in Figure 2. This figure shows the tomograms in velocity coordinates, the locations of the stars, and the predicted gravitational trajectory of the gas stream from the $L_{1}$ point. In addition, the space between the large solid circle and smaller dashed circle represents the locus of a Keplerian disk which extends from the surface of the mass gainer to its Roche lobe in Cartesian space.

The most apparent structure in the tomogram derived from the observed spectra corresponds to the accretion disk surrounding the primary star (Figure 2, top left frame). The emission seen in the observed spectrum is heavily distorted by the underlying spectrum of both stars. The subtraction of the stellar contribution produced a much more pronounced image of the accretion disk (Figure 2, bottom left frame). When the spectra of the stars and disk were removed from the observed spectrum, the tomogram was nearly clear, suggesting that the disk had been successfully modeled. However, when the tomogram was enhanced (Figure 2, top middle frame), it revealed the presence of two weaker features: emission from a gas stream along the predicted gravitational trajectory, and a smaller arc-like structure within the locus of the accretion disk. Finally, when the combined synthetic spectrum of the stars, disk, and gas stream was removed from the observed spectrum, the tomogram was once again clear, but on enhancement (Figure 2, bottom middle frame), the tomogram revealed that the gas stream emission had been adequately modeled. The only remaining structure in the tomogram was the arc-like feature within the accretion disk. This arc was finally understood with the help of hydrodynamic simulations of Richards \& Ratliff (1998) (Figure 2, right frames); it represents the portion of an asymmetric elliptical disk that was not modeled because SHELLSPEC assumes a symmetric circular Keplerian accretion disk (Miller et al. 2007).

The detection of the arc-shaped structure demonstrated for the first time that the accretion disks in Algol systems are asymmetric and elliptical in the velocity domain. Moreover, the isolation of the low-luminosity gas stream in TT Hya also marked the first time that the gas stream had been imaged in this binary. The successful removal of the gas stream emission from the tomogram by Miller et al. (2007) also suggests that the 

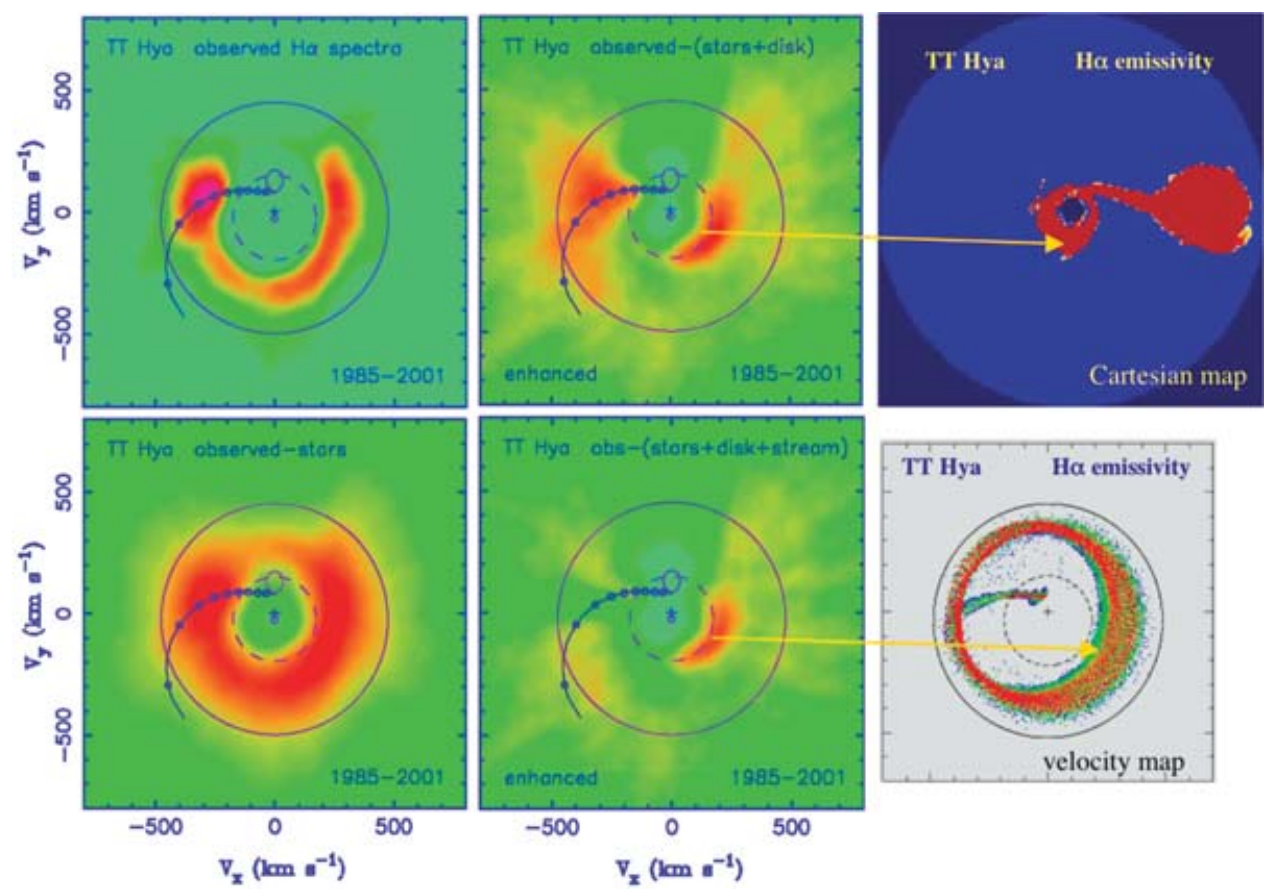

Figure 2. H $\alpha$ Doppler tomograms of TT Hya: (a) the observed spectra (intensity, I $=0.98-$ 1.12), (b) observed spectra minus stars $(\mathrm{I}=0.98-1.6)$, (c) enhanced version of observed spectra minus stars and disk $(\mathrm{I}=0.99-1.2)$, (d) enhanced version of observed spectra minus stars, disk, and gas stream $(\mathrm{I}=0.99-1.2)$; Miller et al. $(2007)$. The eccentric disk in TT Hya was predicted by the hydrodynamic simulations of Richards \& Ratliff (1998).

properties of the gas stream, including the mass accretion rate, can be derived using this technique.

These results demonstrate that the SHELLSPEC code can be used to sequentially model the stars, disk, and gas stream. Only a very faint portion of the disk could not be modeled. Comparisons with simulated $\mathrm{H} \alpha$ tomograms of TT Hya based on hydrodynamic simulations by Richards \& Ratliff (1998) suggested that the unmodeled region was indeed part of an asymmetric accretion disk. This region was not modeled by SHELLSPEC because the code assumes a circular Keplerian disk structure. This result provides the first observational confirmation that the disk in TT Hya is elliptical and asymmetric. Moreover, the similarity between the tomogram based on the observed $\mathrm{H} \alpha$ spectra only and that based on the observed minus stellar contribution suggests that the gas in the accretion disk is optically thin at $\mathrm{H} \alpha$, as assumed in the differencing procedure (see Richards 1993, Richards 2004).

\section{The First 3D Tomograms}

It is well-known that the accretion structures should be fully three dimensional, especially near the hot spot where gas stream comes in contact with the outer edge of the disk (in CVs and long-period Algols) or the actual surface of the mass gainer (in the shortperiod Algols). Evidence of these 3D gas flows is based on studies of ultraviolet spectra and eclipse mapping (see, e.g., Peters 2007, in these proceedings). Nevertheless normal back-projection process translates the images onto the $2 \mathrm{D}$ frame in the orbital plane of the binary. The first 3D tomograms have now been produced by Agafonov et al. (2006) for the short-period Algol system, U CrB. They used a "radioastronomical approach" 


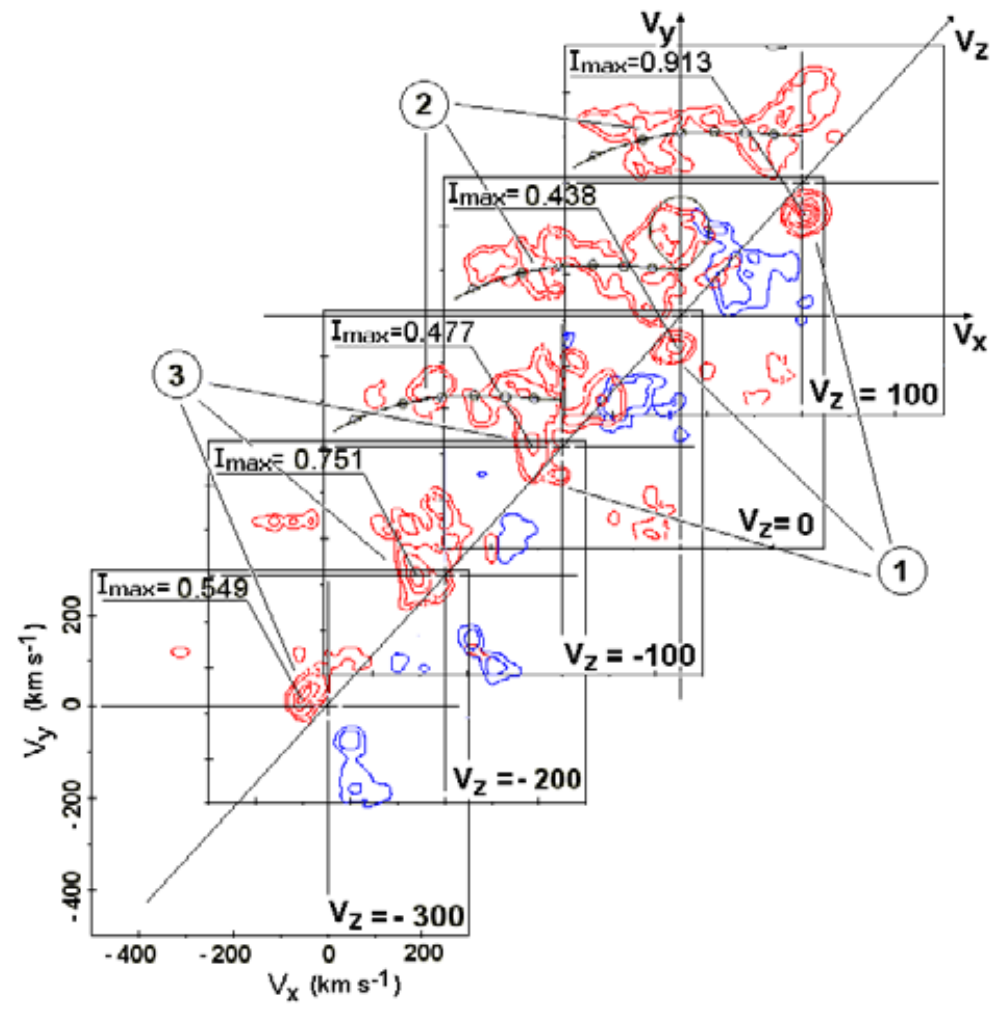

Figure 3. The three-dimensional Doppler tomogram of U CrB from spectra taken in 1994. The three strongest $\mathrm{H} \alpha$ emission features are (1) the accretion annulus, (2) the gas stream moving along the ballistic trajectory from the L1 point, and (3) the high velocity jet moving in the $V_{z}$ direction. (Courtesy: Agafonov et al. 2006).

(RA) which is more effective than the filtered back projection technique, especially under conditions of incomplete orbital coverage resulting in insufficient sampling of the spectra.

The radioastronomical approach is equivalent to the convolution of the unknown 3D Doppler tomogram $f(x, y, z)$ with the summarized point spread function, $h(x, y, z)$ : $g(x, y, z)=f(x, y, z) * * * h(x, y, z)+n(x, y, z)$. Here, the summarized image or "dirty map" is

$$
g(x, y, z)=\sum_{i=1}^{N} u_{i}(x, y, z) \quad \text { and } \quad h(x, y, z)=\sum_{i=1}^{N} h_{i}(x, y, z),
$$

and $n(x, y, z)$ is the noise (Agafonov et al. 2006). In Doppler tomography, the summarized image is constructed by means of back-projecting from the set of line profiles. The function $h(x, y, z)$ is constructed from the set of transfer functions $h_{i}$ by taking into consideration the angles corresponding to the orbital phases and system inclination. These are the same angles used to construct the summarized image.

The 3D tomography of $\mathrm{U}$ CrB using the radioastronomical approach found that the gas flow was typically confined to within $\pm 30 \mathrm{~km} \mathrm{~s}^{-1}$ of the orbital plane. The tomograms also display the first distinct images of the gas stream moving with high velocity $\left(\sim 200 \mathrm{~km} \mathrm{~s}^{-1}\right)$ across the orbital plane of the binary. This is probably a jet of material being ejected from the orbital plane near the splash zone at the star-stream impact site. 


\section{Conclusions}

Doppler tomography has been used successfully to produce images of accretion flows in a variety of interacting binaries including the CVs, X-ray binaries, and Algols. These images would not have been otherwise possible since these systems and too distant to be directly imaged. The accretion structures identified in the compact systems and the longperiod Algols display many similarities, while the short-period Algols present a variety of accretion structures. Miller et al. (2007) have demonstrated that synthetic spectra can be used effectively to extract the properties of the individual contributors to the observed spectra. Their procedure can be used to study other Algol disk systems (e.g., AU Mon). With minor modifications, this approach can also be used to study the disks in novae and cataclysmic variables.

The major highlights of Doppler tomography have been the discovery of spiral structure in the accretion disk (e.g., Steeghs et al. 1997, Harlaftis et al. 1999), the images of gas streams along the predicted gravitational trajectory in the polars and Algols (e.g., Schwope et al. 1997, Richards et al. 1995), and the discovery that at least one Algol disk in not symmetric (Miller et al. 2007). The 3D tomogram produced by Agafonov et al. (2006) using their radioastronomical approach has also revealed, for the first time, an image of gas jets beyond the orbital plane. The application of this technique opens new possibilities for the study of the gas motions beyond the orbital plane in binaries because this provides a clearer understanding of the evolution of close binaries and the physical conditions governing mass transfer.

The new 3D hydrodynamic simulations of Bisikalo \& Matsuda (2007) in these proceedings extends the work of Richards \& Ratliff (1998) to much more exciting directions. There is now the opportunity to compare the hydrodynamic velocity maps with the Doppler tomogram derived from observations to study the flow structure and stream disk impact site more deeply than before. So, this is just the beginning.

\section{Acknowledgements}

I thank my many collaborators on several recent projects related to tomography, especially, Michail Agafonov, Geary Albright, Ján Budaj, Milind Cholkar, Ryuko Hirata, Ilian Iliev, Pavel Koubský, Seiji Masuda, Brendan Miller, Geraldine Peters, Mark Ratliff, Olga Sharova, Vojtěch Simon, and Petr Škoda for their contributions to this review. This research was partially supported by NSF-NATO grant DGE-0312144 and NASA ADP grant NNG04GC48G. I am also grateful for HET observing time from Penn State University. Participation in this symposium was supported by NSF/AAS and IAU travel grants. I dedicate this work to Emilios Harlaftis (1965-2005) who was passionate about Doppler tomography and was a co-discoverer of spiral structure in CV disks.

\section{References}

Agafonov, M.I., Richards, M.T., \& Sharova O.I. 2006, ApJ, 652, 1547

Albright, G.E. \& Richards, M.T. 1996, ApJ, 459, L99

Bisikalo, D.V. \& Matsuda, T. 2007, these proceedings, 356

Budaj, J. \& Richards, M.T. 2004, Contrib. Astron. Obs. Skalnate Pleso, 34, 167

Budaj, J., Richards, M.T., \& Miller B. 2005, ApJ, 623, 411

Groot, P.J. 2001, ApJ, 551, 89

Harlaftis, E., Steeghs, D., Horne, K., Martín, E., \& Magazzú 1999, MNRAS, 306, 348

Herman, G.T. 1980, Image Reconstruction from Projections: The Fundamentals of Computerized Tomography (New York: Academic Press)

Hubeny, I. 1988, Comput. Phys. Comm., 52, 103

Hubeny, I. \& Lanz, T. 1992, A\& A, 262, 501

Hubeny, I. \& Lanz, T. 1995, ApJ, 439, 875 
Hubeny, I., Lanz, T., \& Jeffery, C. S. 1994, Newsletter on Analysis of Astronomical Spectra No.20, C.S. Jeffery (CCP7; St. Andrews: St. Andrews Univ.), 30

Kaitchuck, R.H., Schlegel, E.M., Honeycutt, R.K., Horne, K., Marsh, T.R., White, J.C., \& Mansperger, C.S. 1994, ApJS, 93, 519

Karovska, M. Schlegel, E., Hack, W., Raymond, J. C., \& Wood, B. E. 2005, ApJ, 623, L137-L140

Kempner, J.C. \& Richards, M.T. 1999, ApJ, 512, 345

Kurucz, R.L. 1993, SYNTHE Spectrum Synthesis Programs and Line Data (CD-ROM 18)

Marsh, T. R. 2001, Lecture Notes in Physics, 573, 1

Marsh, T.R. \& Horne, K. 1988, MNRAS, 235, 269

Miller, B., Budaj, J., Richards, M.T., Koubský, P., \& Peters, G.J. 2007, ApJ, 656, 1075

Morales-Rueda, L. 2004, Astron. Nach., 325, 193

Morales-Rueda, L., Marsh, T. R., \& Billington, I. 2000, MNRAS, 313, 454

Peters, G.J. 2007, IAU Symp. 240, W. Hartkopf, E. Guinan, \& P. Harmanec (eds.), in press

Peters, G.J. \& Polidan, R.S. 1998, ApJ, 500, L17

Radon, J. 1917, Berichte Sächsische Akademie der Wissenschaften Leipzig Math. Phys. Kl., 69, 262 (reprinted in 1983: Proc. Symposia Appl. Math, 27, 71)

Richards, M.T. 1993, ApJS, 86, 255

Richards, M.T. 2001, Lecture Notes in Physics, 573, 276

Richards, M.T. 2004, Astron. Nach., 325, 229

Richards, M.T. \& Albright, G.E. 1996, in Stellar Surface Structure, ed. K. Strassmeier \& J. Linsky (Dordrecht: Kluwer), 493

Richards, M.T., Albright, G.E., \& Bowles, L. M. 1995, ApJ, 438, L103

Richards, M.T., Koubský, P., Šimon, V., Peters, G.J., Hirata, R., Škoda, P., \& Masuda, S. 2000, ApJ, 531, 1003

Richards, M.T. \& Ratliff, M.A. 1998, ApJ, 493, 326

Richards, M.T. \& Rosolowsky, E.W. 1998, ASP Conf. Ser., 154, 2038

Robinson, E.L., Marsh, T.R., \& Smak, J.I. 1993, in Accretion Disks in Compact Stellar Systems, ed. J.C. Wheeler (Singapore: World Scientific), 75

Schwope, A.D. 2001, Lecture Notes in Physics, 573, 127

Schwope, A.D., Mantel, K.-H., \& Horne, K. 1997, A\&A, 319, 894

Schwope, A.D., Staude, A., Vogel, J., \& Schwarz, R. 2004, Astron. Nach., 325, 197

Shepp, L.A. 1983, Proc. Symposia in Appl. Math, 27, 1

Sawada, E., Matsuda, T., \& Hachisu, I. 1986, MNRAS, 219, 75

Staude, A., Schwope, A. D., \& Schwarz, R. 2001, A\&A, 374, 588

Steeghs, D. 2004, Astron. Nach., 325, 185

Steeghs, D., Harlaftis, E. T., \& Horne, K. 1997, MNRAS, 290, L28

Vrtilek, S.D., Quaintrell, H., Boroson, B., \& Shields, M. 2004, Astron. Nach., 325, 209

\section{Discussion}

JUAN ECHEVARRIA: A basic assumption of Doppler Tomography is that the accretion material is in the orbital plane. Have you taken into account magnetic fields to explain the partially broken disks you show in some of the systems?

RICHARDS: No, we have not included any treatment of magnetic fields. However, we have checked to see if the magnetic field could have deflected the gas streams from the gravitational path and we plan to add a treatment of magnetic effects in SHELLSPEC, our synthesis code. I should also note, however, that coronal mass ejections (flares) may be the cause of changes in the structure of accretion flows, by changing the mass transfer rate on a time scale of several months.

NiCK ELIAS: How many phases are required to reconstruct an image via Tomography?

RICHARDS: Hundreds would be ideal; 25 is OK if they're evenly spaced around the orbit. 